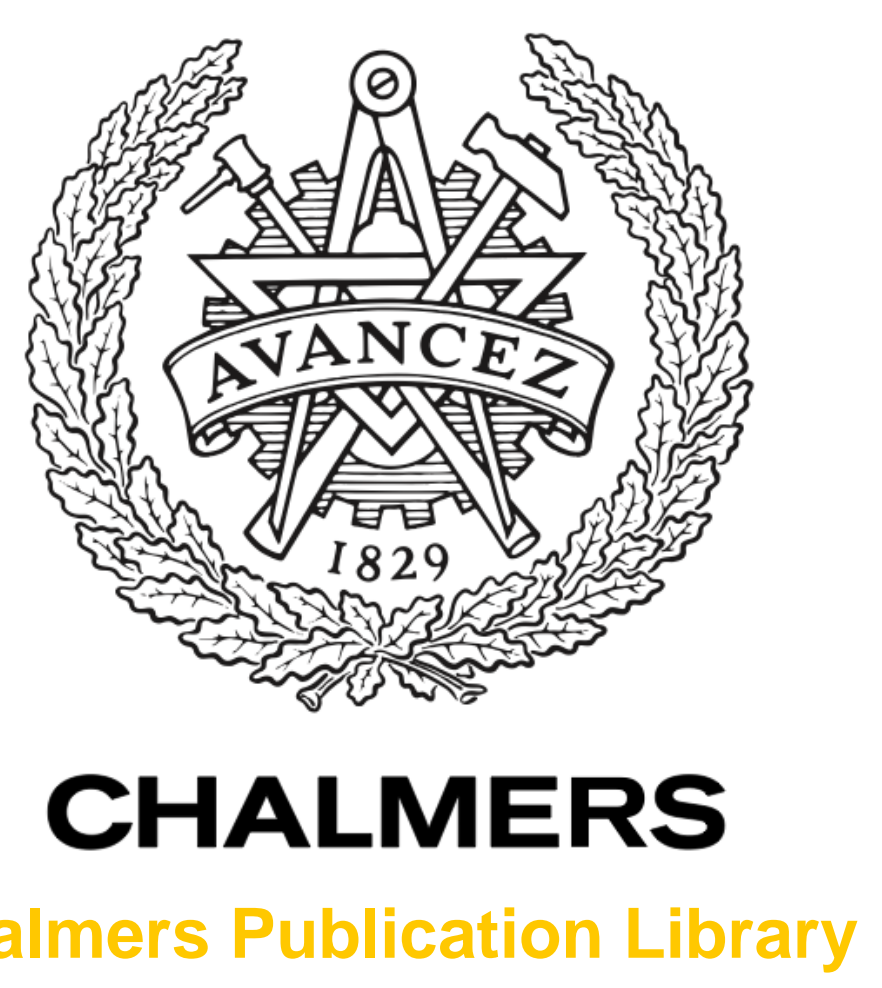

Chalmers Publication Library

\title{
Two-level HARQ for turbo coded cooperation: System retransmission gain and optimal time allocation
}

This document has been downloaded from Chalmers Publication Library $(\mathrm{CPL})$. It is the author's version of a work that was accepted for publication in:

IEEE Wireless Communications and Networking Conference, WCNC 2012, Paris, 1-4 April 2012 (ISSN: 1525-3511)

Citation for the published paper:

Fares, H. ; Graell i Amat, A. ; Langlais, C. (2012) "Two-level HARQ for turbo coded cooperation: System retransmission gain and optimal time allocation". IEEE Wireless Communications and Networking Conference, WCNC 2012, Paris, 1-4 April 2012 pp. 433437.

http://dx.doi.org/10.1109/WCNC.2012.6214405

Downloaded from: http://publications.lib.chalmers.se/publication/163633

Notice: Changes introduced as a result of publishing processes such as copy-editing and formatting may not be reflected in this document. For a definitive version of this work, please refer to the published source. Please note that access to the published version might require a subscription. 


\title{
Two-Level HARQ for Turbo Coded Cooperation: System Retransmission Gain and Optimal Time Allocation
}

\author{
Haïfa Farès ${ }^{\dagger}$, Alexandre Graell i Amat ${ }^{\ddagger}$, Charlotte Langlais ${ }^{\dagger}$, Marion Berbineau ${ }^{\S}$ \\ $\dagger$ Department of Electronics, Institut TELECOM-TELECOM Bretagne, Brest, France \\ $\ddagger$ Department of Signals and Systems, Chalmers University of Technology, SE-412 96 Gothenburg, Sweden \\ $\S$ Université Lille Nord de France, IFSTTAR, LEOST, F-59650 Villeneuve d'Ascq, France \\ Email: \{haifa.fares, charlotte.langlais\}@telecom-bretagne.eu, alexandre.graell@chalmers.se, marion.berbineau@ifsttar.fr
}

\begin{abstract}
Hybrid automatic repeat request (HARQ) is a wellknown technique for improving system throughput and link performance of wireless communication systems, including cooperative communication systems. In this paper, we exploit the limited feedback applied to the two-source turbo coded cooperation scheme to define a particular cooperative HARQ protocol, called two-level HARQ, where the decision on retransmission at each node is conditioned by two levels: first by the feedback from the destination and second by the feedback from the partner node. To evaluate the performance improvement of this cooperative HARQ system over the original turbo coded cooperation system, we define the system retransmission gain. This gain is defined in terms of frame error probability based on the bound on frame error probability. This gain serves as decision parameter to determine conditions under which cooperative HARQ protocol is useful. To implement this adaptive cooperative scheme efficiently, the time resource allocation problem has been explored offering sizable performance improvements.
\end{abstract}

\section{INTRODUCTION}

User cooperation has been proposed as a new alternative used to implement distributed spatial diversity, instead of the original relay channel. In the traditional relaying scheme, where there is a limited number of relay nodes, the sources have to compete with each other by queuing to enjoy the possible diversity gain offered by the relay, since it can assist only one source at a time. However, in multiple-source systems, we can overcome the shortage of relays and consequently we avoid user competition by favoring user cooperation. A practical user cooperation scheme, known as Turbo coded cooperation (TCCoop), was proposed in [1], where distributed turbo coding, cooperation and cyclic redundancy check (CRC) at the partner node were combined. This approach benefits from cooperative diversity, coding and turbo processing gain.

On the other hand, Hybrid Automatic repeat request (HARQ) techniques can be adopted in cooperative wireless networks to overcome throughput degradation due to the fixed cooperative phase and to strengthen the source-to-relay and

This work is partially funded by the PREDIT research project MOCAMIMODYN (MOdèles de CAnaux MIMO DYNamiques en tunnels pour des applications transport public) and the Swedish Agency for Innovation Systems (VINNOVA) under the P36604-1 MAGIC project. source-to-destination links. Several works on HARQ protocols combined with relaying schemes can be found in the literature, e.g., [2-4]. In this paper, we explore cooperative HARQ protocol dedicated to the user cooperation system based on the TCCoop scheme, a configuration that has not yet been fully explored. This particular cooperative scheme named two-level HARQ protocol (TL-HARQ) has been proposed in [5]. The goal of this cooperative HARQ protocol applied to the twosource relay network is twofold: first to increase throughput, by avoiding transmissions when unnecessary; second to improve error rate performance by ensuring more cooperation.

In [6], a frame error probability analysis of the coded cooperation system with convolutional codes based on the pairwise error probability was conducted. The authors defined the cooperation gain to quantify the performance improvement in terms of reliability with respect to the non-cooperative case. Moreover, a geometrical framework was adopted in order to determine conditions under which cooperation is useful and how the channel qualities affect the benefits of cooperation. This paper extends the analysis in [6] to the cooperative HARQ protocol context using turbo processing. We define the system retransmission gain for the TL-HARQ protocol, with respect to the original TCCoop system, in terms of frame error probability. The basis of this performance analysis is the socalled code threshold of a turbo code ensemble, given in [7].

As given in [8], the cooperation level is defined to tune the percentage of incremental redundancy transmitted by the partner, which affects the performance of coded cooperative system in a great degree. In [8], the optimal cooperation level has been determined by minimizing the outage probability of the considered source. In this paper, we determine optimal resource allocation by minimizing the average frame error probability performance of the whole cooperative system. Compared to the outage analysis, our analysis is more complete since it takes into account the used channel code and the efficiency of the proposed TL-HARQ protocol for both cooperating sources. 


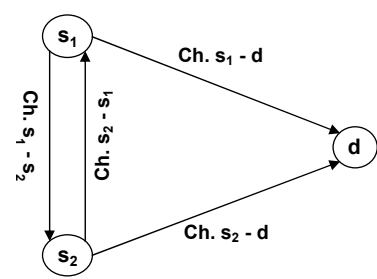

Fig. 1. A three-node cooperative wireless network.

\section{SYSTEM MODEL}

We consider the wireless relay network, depicted in Fig. 1, consisting of two sources $\mathrm{s}_{1}$ and $\mathrm{s}_{2}$, which cooperate to communicate statistically independent data to a single destination d. The network uses the TCCoop strategy and operates in half-duplex mode according to a time-division transmission schedule. It consists in a distributed (over source) turbo code that can be iteratively decoded at the destination. Source $\mathrm{s}_{i}$ $(i=1,2)$ can operate in two different modes: transmission mode, by transmitting its own local information $\left(\mathbf{u}_{i L}\right)$, or relaying mode, by helping the partner node to transmit its information. Both sources are equipped with two encoders $\mathcal{C}_{a}$ and $\mathcal{C}_{b}$ of rates $R_{a}$ and $R_{b}$, respectively, that constitute the elementary encoders of the mother turbo code. In the following, we briefly describe TCCoop when no HARQ is used. Without loss of generality, we focus on the information generated at node $\mathrm{s}_{1}$. The transmission of $\mathbf{u}_{1 L}$, the local data of length $K$ bits, is performed over two time slots, also called phases. In the first phase, source $\mathrm{s}_{1}$ encodes $\mathbf{u}_{1 L}$ by $\mathcal{C}_{a}$ into codeword $\mathrm{x}_{1 L}$, of length $N_{a}=K / R_{a}$ bits. $\mathbf{x}_{1 L}$ is augmented with a CRC and transmitted over the wireless channel. Due to the broadcast nature of the wireless channel both the destination and $\mathrm{s}_{2}$ receive a noisy observation of $\mathrm{x}_{1 L}$. If decoding is successful at $\mathrm{s}_{2}$ (i.e., $\mathrm{s}_{2}$ is able to regenerate $\mathbf{u}_{1 L}$ ), it switches to the relaying mode; in the second phase, $\mathbf{u}_{1 L}$ is first interleaved through an interleaver $\pi$ into $\mathbf{u}_{2 R}=\pi\left(\mathbf{u}_{1 L}\right)$ and then encoded by $\mathcal{C}_{b}$ into $\mathbf{x}_{2 R}$, of length $N_{b}=K / R_{b}$ bits, and forwarded to the destination. On the other hand, if decoding is not successful, $\mathrm{s}_{2}$ operates in the transmission mode (non-cooperative); in the second phase $\mathbf{u}_{2 L}$ is first interleaved by $\pi$ into $\tilde{\mathbf{u}}_{2 L}=\pi\left(\mathbf{u}_{2 L}\right)$ and then encoded by encoder $\mathcal{C}_{b}$ into $\tilde{\mathbf{x}}_{2 L}$ and forwarded to the destination.

With reference to source $s_{1}$ four cases are possible:

- Case $1(\Theta=1)$ decoding at sources $s_{1}$ and $s_{2}$ is successful: two codewords, $\mathbf{x}_{1 L}$ and $\mathbf{x}_{2 R}$, are generated for $\mathbf{u}_{1 L}$. Notice that $\mathbf{x}_{1 L}$ and $\mathbf{x}_{2 R}$ form a codeword of a distributed code, where the first subcodeword is generated by $\mathrm{s}_{1}$ and the second subcodeword is generated by $\mathrm{s}_{2}$, jointly exploited by the destination to estimate $\mathbf{u}_{1 L}$, by iterative decoding.

- Case $2(\Theta=2)$ decoding at sources $s_{1}$ and $s_{2}$ fails: two codewords are transmitted for $\mathbf{u}_{1 L}$, namely $\mathbf{x}_{1 L}$ and $\tilde{\mathbf{x}}_{1 L}$, both generated by $\mathrm{s}_{1}$. Therefore, a distributed (over time) code is obtained. Here, no cooperative diversity is exploited. The overall codeword is $\mathbf{x}=\left(\mathbf{x}_{1 L}, \tilde{\mathbf{x}}_{1 L}\right)$.

- Case $3(\Theta=3)$ decoding at source $s_{1}$ fails, decoding at source $\mathrm{s}_{2}$ is successful: in this case both $\mathrm{s}_{1}$ and $\mathrm{s}_{2}$ dedicate the second phase to transmit $\mathbf{u}_{1 L}$. Therefore, three codewords are generated for $\mathbf{u}_{1 L}: \tilde{\mathbf{x}}_{1 L}, \mathbf{x}_{2 R}$ are first optimally combined before iterative decoding using $\mathbf{x}_{1 L}$.

- Case $4(\Theta=4)$ decoding at source $s_{1}$ is successful, decoding at source $\mathrm{s}_{2}$ fails: a single codeword, $\mathbf{x}_{1 L}$, is allocated to $\mathbf{u}_{1 L}$

We denote by $\alpha=N_{a} / N=N_{a} /\left(N_{a}+N_{b}\right)$ the cooperation level, the ratio of the total channel symbols allocated to the first phase [9].

\section{A. Channel Model}

We denote by $\gamma_{\mathrm{s}_{i} \mathrm{~d}}$ and $\gamma_{\mathrm{s}_{i} \mathrm{~s}_{j}}$ the signal-to-noise ratio $E_{s} / N_{0}$ of the $\mathrm{s}_{i}$-to-d channel and of the $\mathrm{s}_{i}$-to- $\mathrm{s}_{j}$ channel, respectively, where $E_{s}$ is the received signal energy and $N_{0}$ is the single-sided noise power density. All channels are modeled as Rayleigh fading. We assume reciprocal inter-source channels, i.e., $\gamma_{\mathrm{s}_{1} \mathrm{~s}_{2}}=\gamma_{\mathrm{s}_{2} \mathrm{~s}_{1}}=\gamma_{\mathrm{ss}}$. At the destination, the received symbol $y_{i L}^{d}$ from source $s_{i}$ is given by

$$
y_{i L}^{d}=h_{\mathrm{s}_{i} \mathrm{~d}} \bar{x}_{i L}+n_{\mathrm{s}_{i} \mathrm{~d}}
$$

where $\bar{x}_{i L} \in\{ \pm 1\}$ denotes the BPSK modulated symbol of bit $x_{i L}, n_{\mathrm{s}_{i} \mathrm{~d}}$ is the additive white Gaussian noise sample, and $h_{\mathrm{s}_{i} \mathrm{~d}}$ is a zero-mean, circularly symmetric complex Gaussian random variable. We define $\nu_{\mathrm{s}_{i} \mathrm{~d}}=\left|h_{\mathrm{s}_{i} \mathrm{~d}}\right|^{2}$ as the channel power. We consider low-mobility environment, therefore the channel coefficients $h_{\mathrm{s}_{i} \mathrm{~d}}$ are assumed to be constant over the transmission of one frame. This channel model is considered in order to highlight the benefits brought by the cooperative diversity, as opposed to the temporal diversity. This approach was adopted in many previous related works $[2,3]$. In order to take into account the network geometry, the average received SNR per coded bit for the source $s_{i}$ is proportional to $d_{\mathrm{s}_{i} \mathrm{~d}}^{-\beta}$, where $\beta$ is the path loss exponent determined by the environment, and $d_{\mathrm{s}_{i} \mathrm{~d}}$ is the distance between source $s_{i}$ and the destination normalized by a reference distance. We assume that the feedback channels are error free.

\section{Two-LEVEL HARQ PROTOCOL}

Compared to non-cooperation, coded cooperation may suffer from throughput degradation, since two phases are always occupied for a single frame. The second phase brings degradation in throughput if the frames from both sources are correctly decoded after the first phase. In this case, the cooperation phase is not required. Therefore, a first HARQ level is defined at the destination side in order to improve system throughput when frames are correctly decoded. Consequently, the cooperation phase is either canceled (both sources correctly decoded) or adjusted (only one source correctly decoded).

Furthermore, it has been proved that the diversity gain in coded cooperation systems is conditioned by the quality of the inter-source channels [1]. Indeed, diversity gain cannot be obtained unless successful decoding is accomplished at the partner node. An immediate suggestion is to use HARQ protocols to strenghten the inter-source link. Another problem of coded cooperation is that, depending on channel conditions, 
the resulting coding scheme is asymmetric, i.e., it favors one source over the other. Therefore, an additional retransmission decision level, performed at the partner node, can be used to circumvent these two drawbacks. Both feedback messages from the destination and the partner node can be combined to increase the system throughput and to improve error rate performance. The designation two-level HARQ comes from the fact that the protocol works at two levels: First, the destination feeds back ACK or NACK messages to the sources to determine whether the cooperation phase is required or not. The first HARQ level avoids degrading system throughput when frames are correctly decoded. In a second level, if a NACK was received, both sources feed back information on their own decoding to request retransmission from the partner node, if required. The goal of the second HARQ level is to improve the inter-source channels by achieving turbo processing gain at the partner side; and thus to allow a higher degree of cooperation between sources and a higher symmetry. To illustrate the proposed protocol, we detail several possible but not exhaustive cases:

- Case 1 (decoding of both $\mathbf{u}_{1 L}$ and $\mathbf{u}_{2 L}$ is successful at the destination): the destination feedbacks an ACK message to both sources, informing that the cooperation phase is not required, and that transmission of the next information frame can be performed.

- Case $2\left(\mathbf{u}_{1 L}\right.$ is corrupted at both destination and $\left.\mathrm{s}_{2}\right)$ : both the destination and $\mathrm{s}_{1}$ feedback a NACK message regarding $\mathbf{u}_{1 L}$. A retransmission phase is then allocated for $\mathrm{s}_{1}$, which transmits $\tilde{\mathbf{x}}_{1 L}$. $\mathrm{s}_{2}$ attempts to decode $\mathbf{u}_{1 L}$ from $\mathbf{x}_{1 L}$ (from the broadcast phase) and $\tilde{\mathbf{x}}_{1 L}$ (from the retransmission phase) using iterative decoding. The cooperation phase is then performed.

- Case 3 (only decoding of $s_{1}$ fails at the destination after the first phase): no additional information is transmitted for $\mathrm{s}_{2}$. Therefore, $\mathrm{s}_{2}$ does not need its partner cooperation anymore; then, the s1-to-d link will be allocated to $s_{1}$. On the other hand, if possible (depending on CRC), $\mathrm{s}_{2}$ cooperates with $\mathrm{s}_{1}$ by transmitting $\mathrm{x}_{2 R}$.

For more details on the cooperative HARQ protocols, we refer the reader to [5]. Cases 1 and 2 are detailed in Fig. 2.

\section{SySTEM RETRANSMISSION GAIN}

In this section, we first analyze the frame error probability performance of the TCCoop scheme alone and the TL-HARQ protocol dedicated to TCCoop, and then we formalize the concept of the system retransmission gain, to quantify the performance improvement of the TL-HARQ protocol over the original TCCoop scheme.

The frame error probability of the TCCoop system for source $\mathrm{s}_{1}$ can be written as

$$
\begin{aligned}
P_{e, \mathrm{~s}_{1} \mathrm{~d}}^{\mathrm{TCCoop}} & =\left(1-P_{e, \mathrm{~s}_{1} \mathrm{~s}_{2}}^{\varphi_{1}}\right)\left(1-P_{e, \mathrm{~s}_{2} \mathrm{~s}_{1}}^{\varphi_{1}}\right) P_{e, \mathrm{~s}_{1} \mathrm{~d}}^{\varphi_{2}, \Theta=1} \\
& +P_{e, \mathrm{~s}_{1} \mathrm{~s}_{2}}^{\varphi_{1}} P_{e, \mathrm{~s}_{2} \mathrm{~s}_{1}}^{\varphi_{1}} P_{e, \mathrm{~s}_{1} \mathrm{~d}}^{\varphi_{\mathrm{S}_{2}}, \Theta=2} \\
& +\left(1-P_{e, \mathrm{~s}_{1} \mathrm{~s}_{2}}^{\varphi_{1}}\right) P_{e, \mathrm{~s}_{2} \mathrm{~s}_{1}}^{\varphi_{1}} P_{e, \mathrm{~s}_{1} \mathrm{~d}}^{\varphi_{2}, \Theta=3} \\
& +P_{e, \mathrm{~s}_{1} \mathrm{~s}_{2}}^{\varphi_{1}}\left(1-P_{e, \mathrm{~s}_{2} \mathrm{~s}_{1}}^{\varphi_{1}}\right) P_{e, \mathrm{~s}_{1} \mathrm{~d}}^{\varphi_{2}, \Theta=4}
\end{aligned}
$$

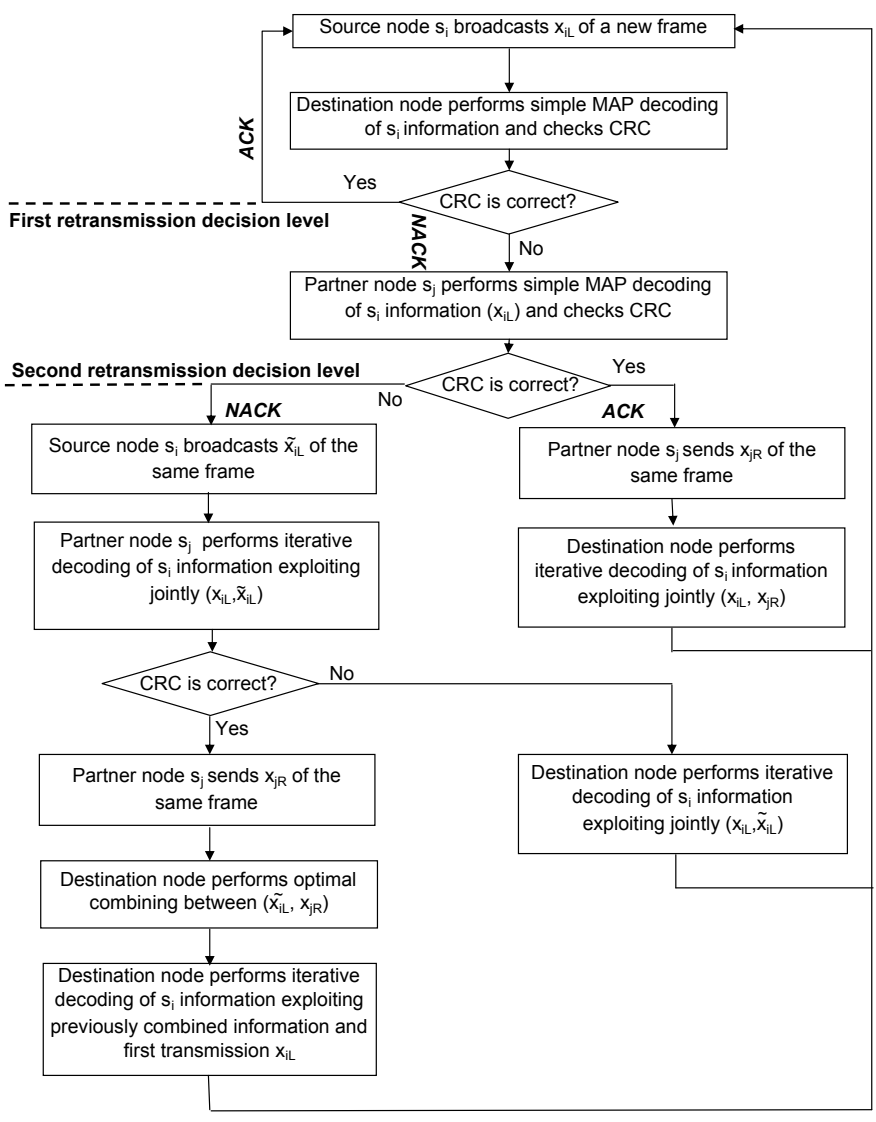

Fig. 2. Simplified flow chart of the proposed TL-HARQ protocol.

where $P_{e, s_{i} \mathrm{~S}_{j}}^{\varphi_{1}}$ denotes the frame error probability of the used channel code (punctured turbo code) over the $\mathrm{s}_{i}$-to- $\mathrm{s}_{j}$ channel and $P_{e, \mathbf{s}_{i} \mathrm{~d}}^{\varphi_{2}, \Theta=k}$ denotes the frame error probability of the transmitted channel code over the $\mathrm{s}_{i}$-to-d channel during both transmission phases, according to the operating case $\Theta=k$.

In [7], it has been showed that, for a turbo code ensemble $[\mathcal{C}]$ transmitted over a Q-block fading channel, where the coefficient is essentially invariant during a single block and different from one block to another, the average maximum likelihood (ML) decoding frame error probability can be bounded as

$$
P_{e}(\bar{\rho}) \leq \operatorname{Pr}\left\{\bar{\rho} \geq \exp \left(-c_{0}\right)\right\}
$$

where $c_{0}$ is the code threshold and $\bar{\rho}$ is the average Bhattacharyya parameter over $\mathrm{Q}$ blocks given by

$$
\bar{\rho}=\sum_{j=0}^{Q-1} \tau_{j} \rho_{j}=\sum_{j=0}^{Q-1} \tau_{j} \exp \left(-\nu_{j} \gamma_{j}\right)
$$

with $\rho_{j}=\exp \left(-\nu_{j} \gamma_{j}\right)$ is the Bhattacharyya parameter over block $j$ and $\tau_{j}$ is the time allocated to the transmission over block $j$.

Using these results, we can now derive a bound on $P_{e, \mathrm{~s}_{i} \mathrm{~s}_{j}}^{\varphi_{1}}$ and $P_{e, s_{i} \mathrm{~d}}^{\varphi_{2}, \Theta=k}$. For instance, after the first transmission phase, decoding of the punctured turbo code with permeability rate $\alpha$ (the ratio between the number of surviving bits and the number of mother code bits) is performed at both sources as well 
as at the destination. The average Bhattacharyya parameter is $\bar{\rho}=\alpha \rho_{\mathrm{s}_{i} \mathrm{~d}}+(1-\alpha) 1$ at the destination. This is given by assuming that the punctured bits are sent to a dummy memoryless channel, whose output is independent of the input, i.e., $\rho_{p}=1$. Consequently, the frame error probability for $\mathrm{s}_{i}$ data (at $\mathrm{s}_{j}$ and at the destination, respectively), can be bounded by

$$
P_{e, \mathrm{~s}_{i} \mathrm{~s}_{j}}^{\varphi_{1}} \leq 1-e^{-c_{0}^{P} \gamma_{\mathrm{s}_{i} \mathrm{~s}_{j}}^{-1}}=\varepsilon_{\mathrm{s}_{i} \mathrm{~s}_{j}}^{\varphi_{1}}, \quad i \neq j
$$

and

$$
P_{e, \mathrm{~s}_{i} \mathrm{~d}}^{\varphi_{1}} \leq 1-e^{-c_{0}^{P} \gamma_{\mathrm{s}_{i} \mathrm{~d}}^{-1}}=\varepsilon_{\mathrm{s}_{i} \mathrm{~d}}^{\varphi_{1}}, \quad i=1,2 .
$$

where $c_{0}^{P}=\log \frac{\alpha}{\exp \left(-c_{0}\right)-(1-\alpha)}$.

By performing the second transmission phase, four cases are possible:

- Case $1(\Theta=1)$ : The whole mother turbo code of source $\mathrm{s}_{1}$ is transmitted over two parallel channels with SNR $\gamma_{\mathrm{s}_{1} \mathrm{~d}}$ and $\gamma_{\mathrm{s}_{2} \mathrm{~d}}$ and with permeability rates $\alpha$ and $1-$ $\alpha$, respectively. The average Bhattacharyya parameter is $\bar{\rho}=\alpha \rho_{\mathrm{s}_{1} \mathrm{~d}}+(1-\alpha) \rho_{\mathrm{s}_{2} \mathrm{~d}}$. Consequently, the frame error probability can be bounded as [7]

$$
\begin{aligned}
P_{e, \mathrm{~s}_{1} \mathrm{~d}}^{\varphi_{2}, \Theta=1} & \leq 1-\omega_{1}-\int_{0}^{-\log \omega_{1}} e^{-\nu_{\mathrm{s}_{1} \mathrm{~d}}} \Upsilon_{1}\left(\nu_{\mathrm{s}_{1} \mathrm{~d}}\right) d \nu_{\mathrm{s}_{1} \mathrm{~d}} \\
& =\varepsilon_{\mathrm{s}_{1} \mathrm{~d}}^{\varphi_{2}, \Theta=1}
\end{aligned}
$$

where

$$
\omega_{1}=\left[\frac{\max \left(e^{-c_{0}}-(1-\alpha), 0\right)}{\alpha}\right]^{\gamma_{\mathrm{s}_{1} \mathrm{~d}}^{-1}}
$$

and

$$
\Upsilon_{1}\left(\nu_{\mathrm{s}_{1} \mathrm{~d}}\right)=\left[\frac{\max \left(e^{-c_{0}}-\alpha e^{-\nu_{\mathrm{s}_{1} \mathrm{~d}} \gamma_{\mathrm{s}_{1} \mathrm{~d}}}, 0\right)}{1-\alpha}\right]^{\gamma_{\mathrm{s}_{2} \mathrm{~d}}^{-1}}
$$

- Case $2(\Theta=2)$ : For this case, where no cooperation is performed, the corresponding frame error probability can be bounded as that of the original turbo code

$$
P_{e, \mathrm{~s}_{1} \mathrm{~d}}^{\varphi_{2}, \Theta=2} \leq 1-e^{-c_{0} \gamma_{\mathrm{s}_{1} \mathrm{~d}}^{-1}}=\varepsilon_{\mathrm{s}_{1} \mathrm{~d}}^{\varphi_{2}, \Theta=2}
$$

- Case $3(\Theta=3)$ : The average Bhattacharyya parameter is $\bar{\rho}=\alpha \rho_{\mathrm{s}_{1} \mathrm{~d}}+(1-\alpha)\left(\rho_{\mathrm{s}_{1} \mathrm{~d}}+\rho_{\mathrm{s}_{2} \mathrm{~d}}\right)$. The frame error probability can be then bounded as

$$
\begin{aligned}
P_{e, \mathrm{~s}_{1} \mathrm{~d}}^{\varphi_{2}, \Theta=3} & \leq 1-e^{-\omega_{2}}-\int_{0}^{\omega_{2}} e^{-\nu_{\mathrm{s}_{1} \mathrm{~d}}} \Upsilon_{2}\left(\nu_{\mathrm{s}_{1} \mathrm{~d}}\right) d \nu_{\mathrm{s}_{1} \mathrm{~d}} \\
& =\varepsilon_{\mathrm{s}_{1} \mathrm{~d}}^{\varphi_{2}, \Theta=3}
\end{aligned}
$$

with

$$
\begin{aligned}
\omega_{2} & =\frac{c_{0}}{\gamma_{\mathrm{s}_{1} \mathrm{~d}}}, \\
\Upsilon_{2}\left(\nu_{\mathrm{s}_{1} \mathrm{~d}}\right) & =\left[\frac{\max \left(e^{-c_{0}} e^{\nu_{\mathrm{s}_{1} \mathrm{~d}} \gamma_{\mathrm{s}_{1} \mathrm{~d}}}-\alpha, 0\right)}{1-\alpha}\right]^{\gamma_{\mathrm{s}_{2} \mathrm{~d}}^{-1}}
\end{aligned}
$$

- Case $4(\Theta=4)$ : The frame error probability of $\mathrm{s}_{1}$, $P_{e, \mathrm{~s}_{1} \mathrm{~d}}^{\varphi_{2}, \Theta=4}$, is then bounded as for $P_{e, \mathrm{~s}_{1} \mathrm{~d}}^{\varphi_{1}}$, given in (6).
By upper bounding the probability of no error by $1-P_{e} \leq 1$, the frame error probability of the TCCoop system for source $\mathrm{s}_{1}$ can be bounded as

$$
\begin{aligned}
P_{e, \mathrm{~s}_{1} \mathrm{~d}}^{\mathrm{TCCoop}} & \leq \varepsilon_{\mathrm{s}_{1} \mathrm{~d}}^{\varphi_{2}, \Theta=1}+\varepsilon_{\mathrm{s}_{1} \mathrm{~S}_{2}}^{\varphi_{1}} \varepsilon_{\mathrm{s}_{2} \mathrm{~s}_{1}}^{\varphi_{1}} \varepsilon_{\mathrm{s}_{1} \mathrm{~d}}^{\varphi_{2}, \Theta=2}+\varepsilon_{\mathrm{s}_{2} \mathrm{~s}_{1}}^{\varphi_{1}} \varepsilon_{\mathrm{s}_{1} \mathrm{~d}}^{\varphi_{2}, \Theta=3} \\
& +\varepsilon_{\mathrm{s}_{1} \mathrm{~s}_{2}}^{\varphi_{1}} \varepsilon_{\mathrm{s}_{1} \mathrm{~d}}^{\varphi_{\varphi_{2}}, \Theta=4}=\varepsilon_{e, \mathrm{~s}_{1} \mathrm{~d}}^{\mathrm{TCC}}
\end{aligned}
$$

For the TL-HARQ protocol several cases must be considered, arising from the decoding results at the destination after the first transmission phase, and, if necessary (unsuccessful decoding), by the decoding results at the partner node. Notice that the additional transmission to the partner node, if requested, is also overheard by the destination node due to the broadcast nature of the wireless link. The bound on the frame error probability of the TL-HARQ protocol for $\mathrm{s}_{1}$ is derived by taking into account all possible transmission cases $(\Theta=1,2,3)$, operating for different conditions, and is given by

$$
\begin{aligned}
P_{e, \mathrm{~s}_{1} \mathrm{~d}}^{\mathrm{TL}} & \leq \varepsilon_{\mathrm{s}_{2} \mathrm{~d}}^{\varphi_{1}}\left[\varepsilon_{\mathrm{s}_{1} \mathrm{~d}}^{\varphi_{2}, \Theta=1}+\varepsilon_{\mathrm{s}_{1} \mathrm{~s}_{2}}^{\varphi_{1}} \varepsilon_{\mathrm{s}_{1} \mathrm{~s}_{2}}^{\varphi_{2}} \varepsilon_{\mathrm{s}_{1} \mathrm{~d}}^{\varphi_{2}, \Theta=2}\right] \\
& +\varepsilon_{\mathrm{s}_{1} \mathrm{~d}}^{\varphi_{2}, \Theta=3}+\varepsilon_{\mathrm{s}_{1} \mathrm{~s}_{2}}^{\varphi_{1}} \varepsilon_{\mathrm{s}_{1} \mathrm{~s}_{2}}^{\varphi_{2}} \varepsilon_{\mathrm{s}_{1} \mathrm{~d}}^{\varphi_{2}, \Theta=3}=\varepsilon_{e, \mathrm{~s}_{1} \mathrm{~d}}^{\mathrm{TL}-\text { ARQ }}
\end{aligned}
$$

where $\varepsilon_{\mathrm{S}_{1} \mathrm{~S}_{2}}^{\varphi_{2}}$ is the bound to the error probability of the mother turbo code transmitted over the $\mathrm{s}_{1}$-to- $\mathrm{s}_{2}$ link (due to the retransmission requested by the partner node).

Notice that for the TL-HARQ protocol, the constraining case $\Theta=4$, where even after all transmission phases, only a punctured turbo code is available at the destination, is avoided.

Definition 1: For a fixed distributed channel code, the system retransmission gain, for the TL-HARQ protocol, is defined as

$$
G_{f, \mathrm{TL}-\mathrm{HARQ}}^{\mathrm{Rtx}}=\frac{\varepsilon_{e, \mathrm{~s}_{1} \mathrm{~d}}^{\mathrm{TCCop}}+\varepsilon_{e, \mathrm{~s}_{2} \mathrm{~d}}^{\mathrm{TCCoo}}}{\varepsilon_{e, \mathrm{~s}_{1} \mathrm{~d}}^{\mathrm{TL} d}+\varepsilon_{e, \mathrm{~s}_{2} \mathrm{~d}}^{\mathrm{TL}-\mathrm{dRQ}}} .
$$

\section{NumericAl Results}

In Fig. 3, frame error rate (FER) simulation results as well as bounds on the frame error probability are given as functions of $\gamma_{\mathrm{sd}}^{b}$ (symmetric uplink case) where $\gamma_{\mathrm{sd}}=\gamma_{\mathrm{ss}}$. For simulation results, we consider the rate- $1 / 2$ convolutional encoder with generator polynomials $(1,15 / 13)_{8}$ in octal form for $\mathcal{C}_{a}$ and the rate- 1 convolutional encoder with generator polynomial $(17 / 13)_{8}$ for $\mathcal{C}_{b}$. The information block length is $K=128$ bits and free space environment is assumed $(\beta=2)$. For fair comparisons, all results in this section are given in terms of $\gamma^{b}$, where $\gamma^{b}=\gamma \bar{R}, \bar{R}$ being the average rate of the system. Note that the average rate of the system depends on the code rate and on the number of retransmission attempts. We obtain the non-surprising result that the frame error probability bound curves are parallel to the simulated FER curves. The bounds on the frame error probability can therefore be adopted as a starting point for analytical guidelines. Results are given for two different time allocation scenarios:

- $\alpha=2 / 3$ : the optimal time allocation of the coded cooperation scheme in terms of outage probability for this particular channel conditions, given in [8]. 


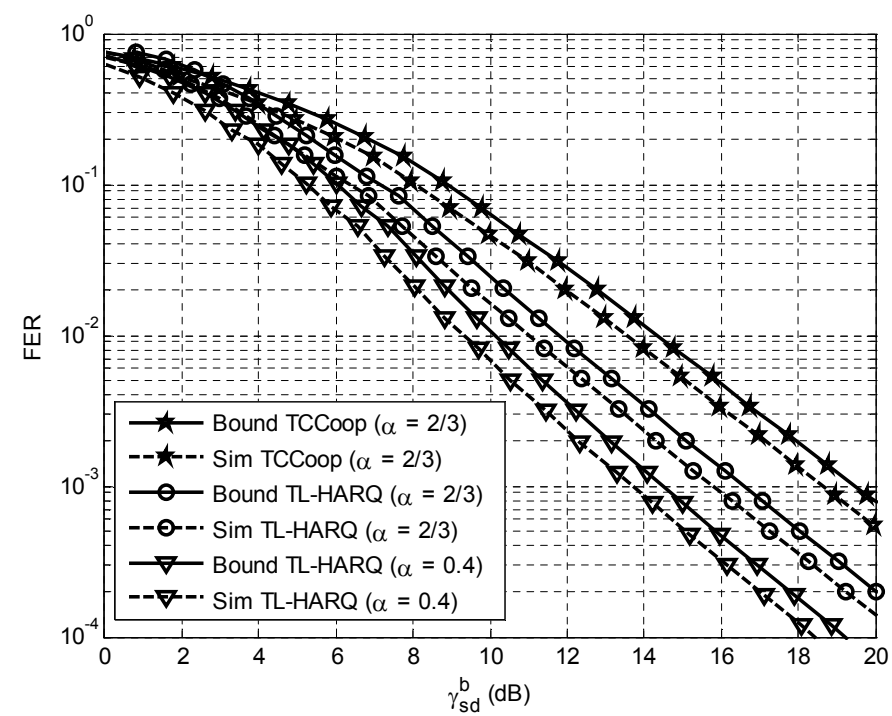

Fig. 3. Bounds on the frame error probability and FER simulation for $\alpha=$ $2 / 3$ versus $\alpha_{\text {opt }}$ for equal channel conditions $\gamma_{\mathrm{sd}}=\gamma_{\mathrm{ss}}$

- $\alpha=0.4$ : the optimal time allocation of the TL-HARQ protocol determined by minimizing numerically the average frame error probability performance of the whole cooperative system for these channel conditions, i.e., $\alpha_{\text {opt }}=\operatorname{argmin}_{\alpha} \varepsilon_{e, \mathrm{~s}_{1} \mathrm{~d}}^{\mathrm{TL}-\mathrm{H} Q}+\varepsilon_{e, \mathrm{~s}_{2} \mathrm{~d}}^{\mathrm{TL}-\mathrm{d} Q}$.

We note that the TL-HARQ protocol improves the reliability performance of the TCCoop scheme, for $\alpha=2 / 3$, by achieving higher diversity gain, ensuring more cooperation between the sources and better overall transmission energy by avoiding unnecessary transmissions. However, more improvements are possible when an efficient time allocation between different transmission phases is performed.

In the following, we determine the geometric conditions under which using TL-HARQ protocol is useful. We consider a three-node system where the source $s_{1}$ is fixed and $s_{2}$ is moving on the same line from $\mathrm{s}_{1}$ towards the destination. Taking into account the path loss effect, the received SNRs of the $\mathrm{s}_{1}$-to-d, $\mathrm{s}_{1}$-to-s $\mathrm{s}_{2}$ and $\mathrm{s}_{2}$-to-d channels are given by $\left(E_{s} / N_{0}\right),\left(E_{s} / N_{0}\right) d^{-\beta}$ and $\left(E_{s} / N_{0}\right)(1-d)^{-\beta}$, respectively, where $d$ is the normalized inter-source distance (normalized by $d_{\mathrm{S}_{1} \mathrm{~d}}$ ). In Fig. 4, we examine the frame error probability retransmission gain of TL-HARQ as a function of $d$, for a fixed $\gamma_{\mathrm{s}_{1} \mathrm{~d}}^{b}=5 \mathrm{~dB}$ and two time allocation scenarios $(\alpha=2 / 3$ and $\alpha_{\text {opt }}$ ). Regardless the location of $\mathrm{s}_{2}$, the TL-HARQ protocol benefits to the whole cooperative system with respect to the TCCoop scheme, from frame error probability perspective. Its yields to sizable retransmission gains for $\alpha_{\text {opt }}$. We notice also that the best partner location depends on the value of $\alpha$. Nevertheless, the best reliability behavior is always obtained when $s_{2}$ is much closer to the destination: since another retransmission phase to the partner node is possible, the intersource link becomes less critical.

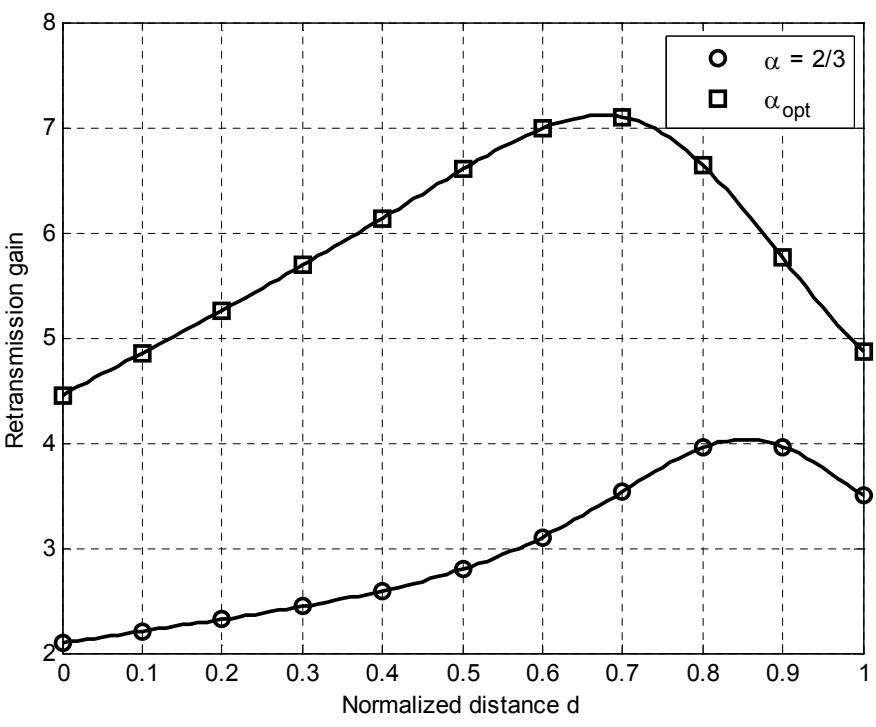

Fig. 4. System retransmission gain over the linear scenario for $\alpha=2 / 3$ versus $\alpha_{\mathrm{opt}}\left(\gamma_{\mathrm{sd}}^{b}=5 \mathrm{~dB}\right)$.

\section{CONCLUSIONS}

In this paper, we we derived frame error probability bound for the TCCoop and its dedicated HARQ protocol called twolevel HARQ. From this bound the optimal time allocation for the TL-HARQ is computed. Finally, we defined the system retransmission gain in order to quantify the benefit of retransmission for this two user cooperation scheme based on the TCCoop scheme. We showed that, while avoiding extensive computations, this analytical performance metric can be adopted as a decision parameter to determine geometric conditions where performing this protocol is useful.

\section{REFERENCES}

[1] M. Janani, A. Hedayat, T. E. Hunter, and A. Nosratinia, "Coded cooperation in wireless communications: space-time transmission and iterative decoding," IEEE Trans. Signal Processing, vol. 52, pp. 362-371, Feb. 2004.

[2] E. Zimmermann, P. Herhold, and G. Fettweis, "The impact of cooperation on diversity-exploiting protocols," in Proc. IEEE Vehicular Technology Conference (VTC), pp. 410-414, May 2004.

[3] Y. Nam, K. Azarian, H. E. Gamal, and P. Schniter, "Cooperation through ARQ," in IEEE Proc. on Signal Processing Advances in Wireless Communications (SPAWC), pp. 1023-1027, June 2005.

[4] I. Stanojev, O. Simeone, Y. Bar-Ness, and D. Kim, "Energy efficiency of non-collaborative and collaborative hybrid-arq protocols," IEEE Trans. Wireless Commun., vol. 8, pp. 326-335, Jan. 2009.

[5] H. Farès, C. Langlais, A. Graell i Amat, and M. Berbineau, "Two-level HARQ for turbo coded cooperation," in Proc. IEEE Vehicular Technology Conference (VTC), pp. 1-5, May 2010.

[6] Z. Lin, E. Erkip, and A. Stefanov, "Cooperative regions and partner choice in coded cooperative systems," IEEE Trans. Commun., vol. 54, pp. 1323 1334, July 2006.

[7] R. Liu, P. Spasojevic, and E. Soljanin, "Incremental redundancy cooperative coding for wireless networks: Cooperative diversity, coding, and transmission energy gains," IEEE Trans. Inf. Theory, vol. 54, pp. 12071224, Mar. 2008.

[8] T. Hunter, S. Sanayei, and A. Nosratinia, "Outage analysis of coded cooperation," IEEE Trans. Inf. Theory, vol. 52, pp. 375-391, Feb. 2006.

[9] T. Hunter and A. Nosratinia, "Diversity through coded cooperation," IEEE Trans. Commun., vol. 5, pp. 283-289, Feb. 2006. 